\title{
Ultrasonographic identification of the anatomical landmarks that define cervical lymph nodes spaces
}

\section{Lavinia Manuela Lenghel' ${ }^{1}$, Grigore Baciuț ${ }^{2}$, Carolina Botar-Jid $^{1}$, Dan Vasilescu ${ }^{1}$, Anca Bojan $^{3}$, Sorin M. Dudea ${ }^{1}$}

${ }^{1}$ Radiology Department, ${ }^{2}$ Cranio-Maxillo-Facial Surgery Department, ${ }^{3}$ Haematology Department, "Iuliu Haţieganu" University of Medicine and Pharmacy, Cluj-Napoca, Romania

\begin{abstract}
The localization of cervical lymph nodes is extremely important in practice for the positive and differential diagnosis as well as the staging of cervical lymphadenopathies. Ultrasonography represents the first line imaging method in the diagnosis of cervical lymphadenopathies due to its excellent resolution and high diagnosis accuracy. The present paper aims to illustrate the ultrasonographic identification of the anatomical landmarks used for the definition of cervical lymphatic spaces. The application of standardized views allows a delineation of clear anatomical landmarks and an accurate localization of the cervical lymph nodes.
\end{abstract}

Keywords: ultrasonography, lymph nodes, lymphadenopathy, cervical region

\section{Introduction}

Ultrasonography (US) represents the first line imaging method used for the assessment of pathological alterations of the head and neck region. A major advantage of the ultrasonographic examination of the neck is represented by its excellent resolution in the exploration of superficial structures, US being able to visualize even non palpable nodules.

The practical value of using ultrasonography lies in its capacity to distinguish benign adenopathies from malignant ones, as well as in its ability to accurately, topographically, localize pathological lymph nodes.

Being able to appoint metastatic lymph nodes to a specific lymph node group is especially important in establishing the stage and prognosis of cervical malignancies, while it is possible even to suggest the site of the

Received 10.12.2012 Accepted 29.12.2012

Med Ultrason

2013, Vol. 15, No 1, 29-34

Corresponding author: Lavinia Manuela Lenghel

Radiology Department,

"Iuliu Hatieganu" University of Medicine and Pharmacy

1-3 Clinicilor str,

400006, Cluj-Napoca, Romania

E-mail: manu_2416@yahoo.com primary lesion. In this regard it is important both to analyze the semiologic criteria that allow the differentiation between benign and malignant as well as to sonographically identify the anatomical landmarks that help decide the space to which the visualized lymph nodes belong [1].

The present paper aims to present the basic anatomical aspects that define and rule the physiology of the cervical region lymphatics and to present the distribution of the cervical lymph nodes on different levels. The main purpose of the paper is to illustrate the ultrasonographic landmarks that allow the placement of a lymph node in a certain cervical space.

\section{Anatomy and physiology}

The lymphatic system consists of: lymph fluid, lymphocytes, lymphatic vessels and lymphatic organs (represented by lymph nodes, thymus, spleen). This system has three main roles: it maintains the balance of the fluids within the tissues, represents the main way of lipid absorption within the intestinal tract, and, last but not least, it plays a major role in body immunity.

The diffuse lymphatic tissue does not have a clear delineation, but it is represented by lymphocytes that are spread under mucosal membranes and around lymphatic nodules. 
Lymphatic capillaries, which unlike blood capillaries do not have a basement membrane, join together to form larger lymphatic vessels. These vessels have valves that direct the lymphatic circulation in a one-way, centripetal, manner. The central nervous system, the bone marrow and the non-vascularized tissues (cornea, epidermis and cartilage) have no lymphatic capillaries. The lymph fluid is filtered through at least one lymph node before it enters the blood flow. The lymph from the upper limb and the right half of the head and neck is drained into the right lymphatic duct, which enters the right subclavian vein. The lymph from the rest of the body is collected into the thoracic duct which then flows into the left subclavian vein. The thoracic duct originates in the superior abdomen from cisterna chyli [2].

The lymph nodes are incapsulated, ovoid, 1 to 25 $\mathrm{mm}$ diameter anatomical structures, located along the lymphatic vessels. The internal architecture of the lymph nodes consists of an outer cortex (located under the capsule and made of lymphatic follicles) and an inner medulla (situated in the centre and consisting of lymphatic tissue conglomerates). These two regions are separated by fibrous trabeculae which originate in the conjunctive capsule. The lymph enters the node through the afferent vessels and flows from the cortex to the medulla, reaches the hillum and then leaves it through the efferent vessels. These represent the afferent vessels for other lymph nodes or enter the thoracic duct or the right lymphatic duct $[2,3]$. The hillum contains the lymph node vessels: the afferent artery and the efferent vein. The paracortex, an intermediary area between the cortex and the medulla, represents a transition area [3]. The structure of the

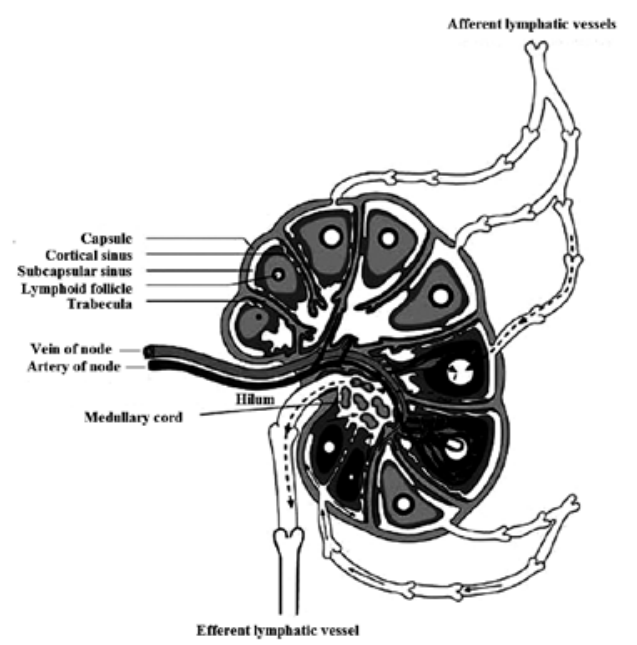

Fig 1. The structure of the lymph nodes, inspired and modified after Ying $M$ and Ahuja AT [3] lymph nodes, illustrated in figure 1, was inspired and modified after Ying M and Ahuja AT [3].

\section{Localization of cervical lymph nodes}

There are two types of classifications used to localize the cervical lymph nodes: topographical (which uses the system of the cervical triangles) and surgical (that uses the criteria of the levels).

The present paper proposes the surgical classification, which uses the criteria of the levels. Ruggierro et al [4] introduced a classification developed and used by the Memorial Sloan Kettering Cancer Center in order to describe the patterns of metastatic dissemination as they were observed on a number of over 1000 patients with radical cervical dissection. The lymph nodes are grouped in six levels, noted with Roman numerals from I to VI, as seen in table I.

The levels are illustrated in figure 2, which was inspired and modified after Ruggiero FP [4].

Table I. The cervical lymph nodes levels

\begin{tabular}{|c|c|c|}
\hline Level I & $\begin{array}{l}\text { I A } \\
\text { I B }\end{array}$ & $\begin{array}{l}\text { Submental nodes } \\
\text { Submandibular nodes }\end{array}$ \\
\hline Level II & $\begin{array}{l}\text { II A } \\
\text { II B }\end{array}$ & $\begin{array}{l}\text { Antero-inferior upper jugular nodes } \\
\text { Postero-superior upper jugular nodes }\end{array}$ \\
\hline Level III & & Middle jugular nodes \\
\hline Level IV & & Lower jugular nodes \\
\hline Level V & $\begin{array}{l}\text { V A } \\
\text { V B }\end{array}$ & $\begin{array}{l}\text { Superior posterior neck group } \\
\text { Inferior posterior neck group }\end{array}$ \\
\hline Level VI & & Central (anterior) group \\
\hline
\end{tabular}

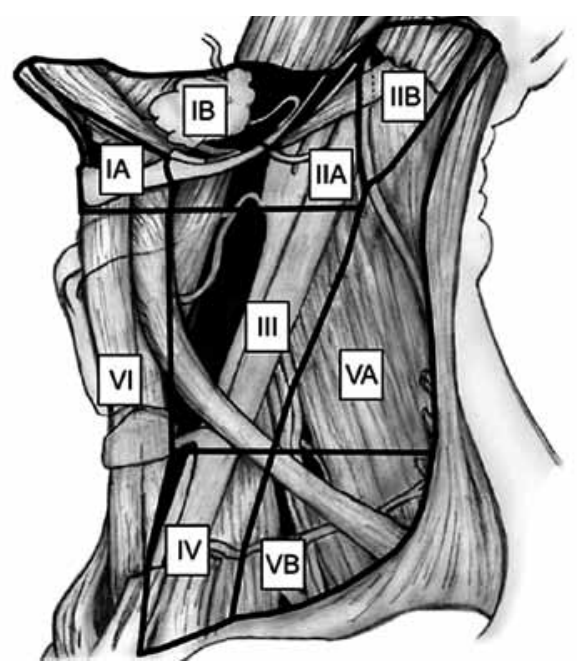

Fig 2. Illustration of the levels, inspired and modified after Ruggiero FP [4] 


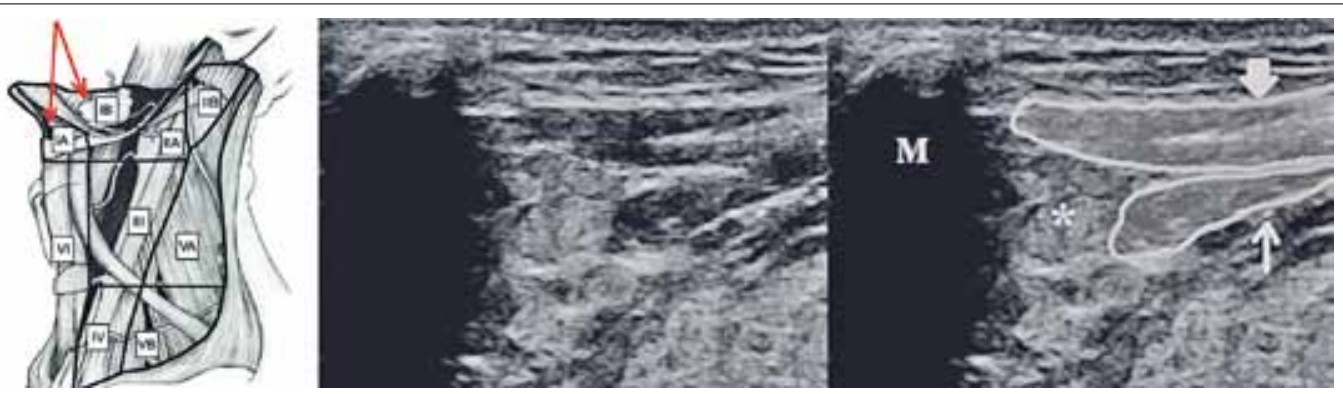

Fig 3. Illustration of the limits of level I: body of the mandible (M) superiorly and the anterior belly of the digastric muscle visualized anteriorly. The asterix $(*)$ indicates the submadibular gland.
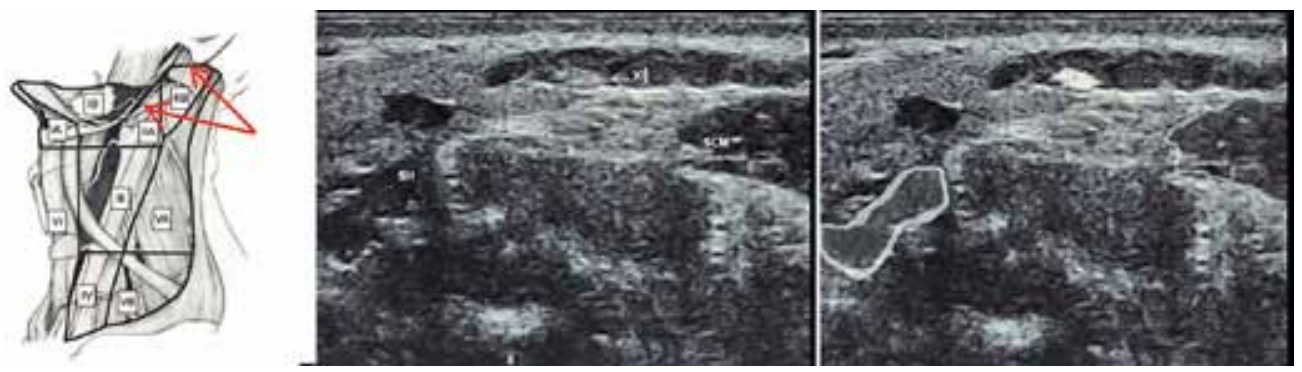

Fig 4. Illustration of the limits of level II: anterior is the stylohyoid muscle (SH), posteriorly the posterior margin of the sternocleidomastoid muscle (SCM) is visualized and between the two anatomical elements can be identified the accessory spinal nerve (XI)

The ultrasonographic identification of the anatomical landmarks that define the cervical lymphatic spaces was based on transverse and longitudinal scans and panoramic images acquired on a HITACHI EUB 8500, using 6.5-13 MHz linear transducer. The examination was performed with the patient supine and head in extension. In order to obtain the head extension a pillow was placed under the patient's shoulders.

Level I (fig 3): the upper limit is represented by the body of the mandible, the posterior by the stylohyod muscle and anterior by the anterior belly of the digastric muscle. This level is divided into two regions:

- Level Ia (submental) is situated between the anterior belly of the digastric muscle and the hyoid bone; tumors of the mouth floor, of the anterior region of the tongue, the alveolar margin of the mandible and inferior lip frequently disseminate at this level.

- Level Ib (submandibular) is located posterior and lateral from the anterior belly of the digastric muscle; at this level metastatic lymph nodes from oral cavity, anterior nasal cavity, soft structures of the face and submandibular gland tumors are often encountered.

Close to the level I lymph node group are located the perifacial (adjacent to the facial vascular bundle, above the mandible margin) and the buccinator lymph nodes.
On the ultrasound images acquired in a transverse plane with the horizontal ramus of the mandible, the body of the mandible superiorly and the anterior belly of the digastric muscle visualized anteriorly can be identified.

Level II (fig 4) is delineated anteriorly by the stylohyoid muscle and posteriorly by the posterior margin of the sternocleidomastoid muscle. This group is situated close to the superior part of the jugular vein, extending from the base of the skull towards the inferior margin of the hyoid bone. In this lymphatic space usually are metastasizing tumors of the oral cavity, nasal cavity, nasopharynx, oropharynx, hypopharynx, larynx and parotid gland. This level is divided by the accessory spinal nerve into:

- Level II a located anterior, inferior and closer to the internal jugular vein

- Level II b situated above and behind the nerve

On the transverse ultrasonographic images these anatomical landmarks can be identified as follows: anterior is the stylohyoid muscle, posteriorly the posterior margin of the sternocleidomastoid muscle is visualized and between the two anatomical elements can be identified the accessory spinal nerve, the one responsible for the division of this level. 

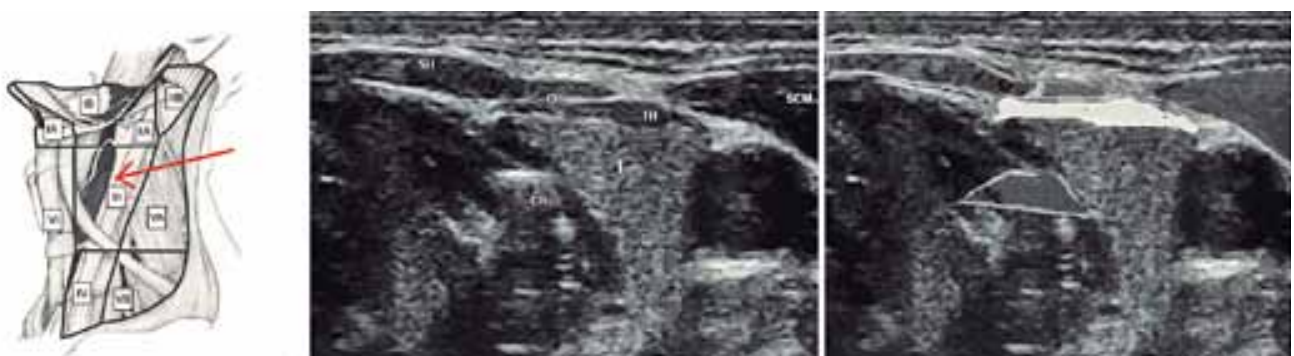

Fig 5. Illustration of the limits of level III: sternohyoid muscle ( $\mathrm{SH})$ anteriorly, the posterior margin of the sternocleidomastoid muscle (SCM) posteriorly and the cricoid cartilage (CR) inferiorly. The omohyoid muscle $(\mathrm{OH})$ visualized anteriorly and the thyrohyoid muscle $(\mathrm{TH})$ situated posteriorly from the omohyoid and anteriorly from the thyroid are also easily seen.
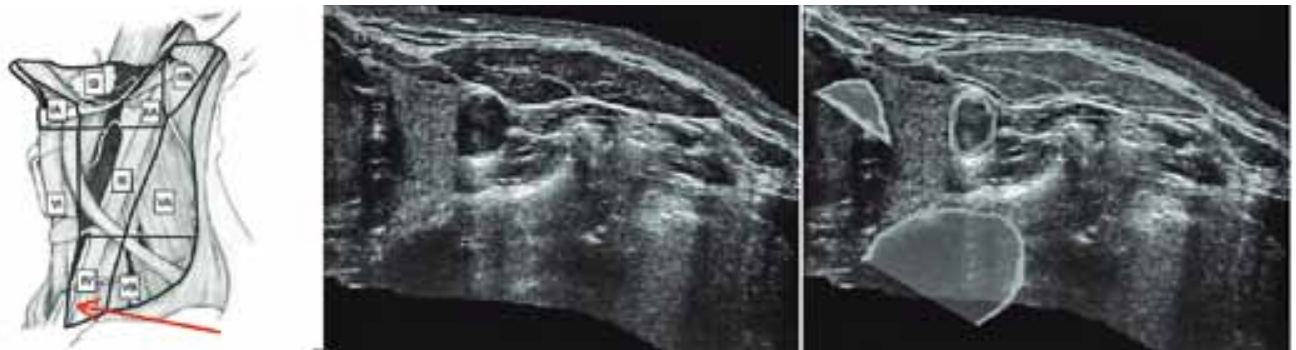

Fig 6. Illustration of the limits of level IV: superiorly the cricoid cartilage (CR), anteriorly the sternohyoid muscle (SH), posteriorly the posterior margin of the sternocleidomastoid muscle (SCM), inferiorly the clavicle $(\mathrm{CL})$

Level III (fig 5) is situated above the hyoid bone, under the inferior margin of the cricoid cartilage, anterior from the sternohyoid muscle and posterior to the posterior margin of the sternocleidomastoid muscle. Tumors from the oral cavity, nasopharynx, oropharynx, hypopharynx and larynx most often disseminate in this lymphatic space.

On the sonographic images performed on the transverse plane the sternohyoid muscle is identified anteriorly, the posterior margin of the sternocleidomastoid muscle posteriorly and the cricoid cartilage inferiorly. The omohyoid muscle visualized anteriorly and the thyrohyoid muscle situated posteriorly from the omohyoid and anteriorly from the thyroid are also easily seen.

Level IV (fig 6) is situated between the inferior margin of the cricoid cartilage (superior), the clavicle (inferior), the sternohyoid muscle (anterior) and the posterior margin of the sternocleidomastoid muscle (posterior). Lymph node metastases of this group most frequently develop from the larynx, hypopharynx, thyroid gland and the cervical segment of the esophagus tumors. The lymph nodes adjacent to the inferior third of the jugular vein are situated at this level.

On the transverse ultrasound images can by identified the following: superiorly the cricoid cartilage, ante- riorly the sternohyoid muscle, posteriorly the posterior margin of the sternocleidomastoid muscle, inferiorly the clavicle. At this level the thyroid lobes and the common carotid artery are also found.

Level V (fig 7) comprises the lymph nodes located in the posterior triangle of the neck, which includes the accessory spinal group, the transverse cervical group and the supraclavicular group. The limits are represented by the posterior margin of the sternocleidomastoid muscle anteriorly, the anterior margin of the trapezius muscle posteriorly, the apex of the sternocleidomastoid and trapezius muscles superiorly and the clavicle inferiorly. This level is divided by a plane represented by the inferior margin of the cricoid cartilage into:

- Level Va (superior): the lymph nodes of the accessory spinal group

- Level Vb (inferior): the transverse and supraclavicular group

In this space are usually encountered metastases from the nasopharynx, oropharynx and the skin of the posterior region of the scalp and neck.

The ultrasonographic images obtained in a transverse view identify the cricoid cartilage superiorly, the posterior margin of the sternocleidomastoid muscle anteriorly, 

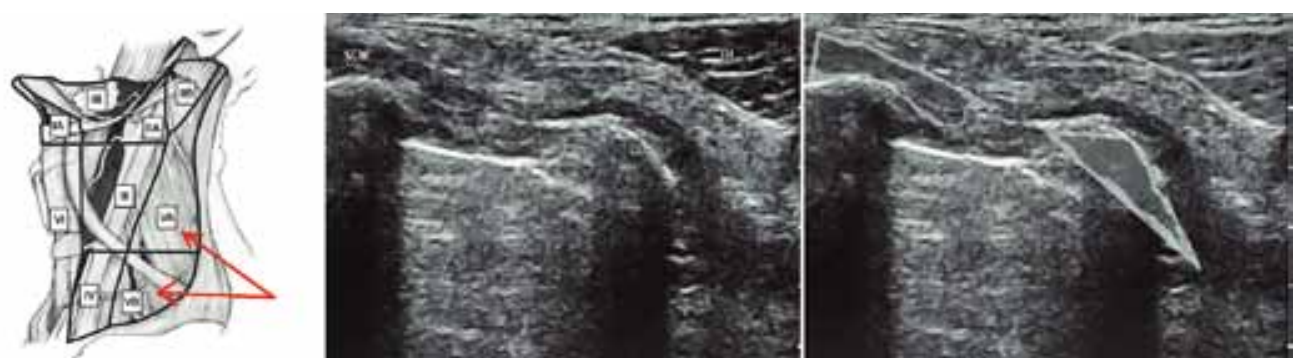

Fig 7. Illustration of the limits of level V: the cricoid cartilage (CR) superiorly, the posterior margin of the sternocleidomastoid muscle (SCM) anteriorly, the trapezius muscle (TR) posteriorly and the clavicle (CL) inferiorly
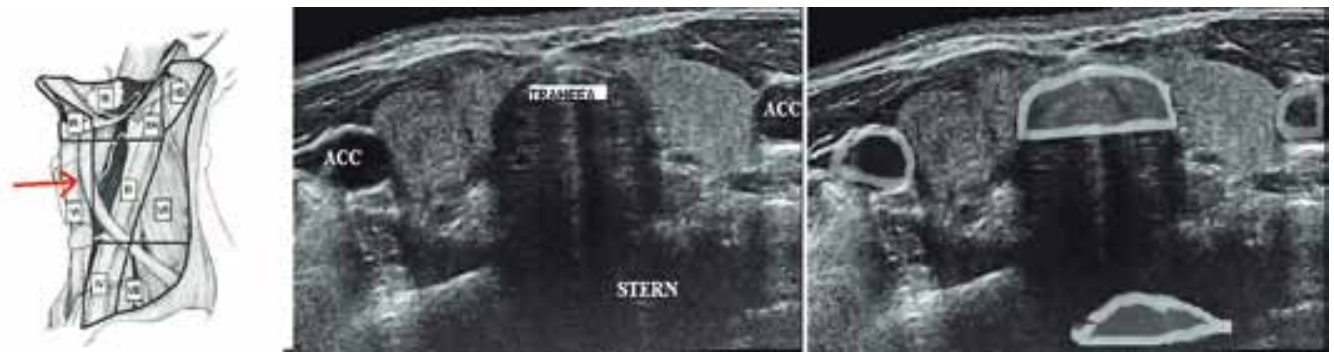

Fig 8. Illustration of the limits of level VI: carotid arteries (ACC) which mark the lateral margins of this level, the trachea (TRAHEEA) is situated superiorly, the sternal notch (STERN) defining the inferior margin.

the trapezius muscle posteriorly, and the clavicle inferiorly.

Level VI (fig 8) is located between the carotid arteries (laterally), the hyoid bone (superiorly) and the sternal notch (inferiorly). The lymph nodes are found in the tracheoesophageal groove (paratracheal), in front oh the trachea (pretracheal), around the thyroid (parathyroid) and on the cricothyroid membrane (precricoid or Delphian). Thyroid tumors, tumors of the subglottic larynx, tracheal (cervical portion), hypopharynx and cervical esophagus tumors usually metastasize at this level.

In order to identify the anatomical elements of this level a panoramic image was acquired, that allows the visualization of both carotid arteries which mark the lateral margins of this level. The trachea is situated superiorly, while the sternal notch defines the inferior margin of level VI.

\section{Discussions}

Ultrasonography is the imaging method most frequently used to evaluate the cervical region. For an accurate examination of this region it is necessary that the examiner has good knowledge of the anatomy of the region, has information about the clinical status of the patient and is familiar with the ultrasonographic pathological aspects [5].

The ultrasonographic examination of the cervical lymph nodes follows a complete clinical exam that evaluates all cervical organs and structures: salivary glands, thyroid, muscle structures, etc. The clinical exam does not allow the identification of the lymph nodes which are less than $5 \mathrm{~mm}$ in diameter, even if they are superficial $[1,5]$.

Ultrasonography has high sensitivity and specificity in the evaluation of head and neck adenopathies. CT has lower sensitivity and specificity, especially in describing the shape, delineation and structure of small size lymph nodes or those situated close to structures that produce artifacts (mandible, dental implants, osteosynthesis plates). The MRI exam presents the same limitations, the two methods being superior in the evaluation of adenopathies found deep into the cervical region [1].

For almost four decades, the classification that was used most frequently was the one proposed by Rouvière in 1938. His paper defines a precise, anatomical, localization of the lymph nodes and of the lymphatic drainage areas in nodes groups. The landmarks used in this classical 
description of the lymph nodes were the ones identified through palpation and the ones observed during surgery and dissection by the author [6].

In 1981 Shah et al [7] proposed that anatomical classification be replaced by a more simple one based on levels. Since then, several classifications were proposed using the level, the region or the area as reference. The purpose of these classifications was to group the cervical lymph nodes based on the clinical and physiopathological available data. The objective of the classification changed from a purely anatomical study to a mapping guide in order to select the most appropriate surgical technique out of the various possible cervical dissections.

The AJCC classification (American Joint Committee on Cancer and the American Academy of Otolaryngology-Head and Neck Surgery) of the cervical lymph nodes introduced in 1991, modified in 1992 and reviewed in 2008, proposes 7 levels, noted with Roman numerals from I to VII.

Some lymph node groups were not included (parotid, retropharingeal), while others are difficult to visualize through ultrasonography (prelaringeal, paratracheal and upper mediastinal). That is why this classification is the most frequently used for CT and MRI examinations [8]. For the ultrasonographic exam Hajek et al. [9] proposed in 1986 another classification, with 8 regions depending on their cervical localization, using the criteria of the neck triangles. In this classification the following triangles are found: submental, submandibular, parotid, upper cervical (above the hyoid, along the internal jugular vein), middle cervical (between the hyoid bone and the cricoid cartilage, along the internal jugular chain), lower cervical (under the cricoid cartilage, along the along the internal jugular chain), supraclavicular and posterior (accessory chain)

The surgical classification proposed by Ruggierro et al and belonging to the Memorial Sloan Kettering Cancer Center is the one that is used most often and it describes the patterns of metastatic dissemination observed in over 1000 patients which underwent radical cervical dissection [4].

Along the studies that propose and describe the two classifications, there are several publications in literature where can be found anatomical and technical data about the elements that participate in the delineation of the cervical lymphatic spaces, which have an increased signifi- cance in establishing the localization and the group the nodes belong to $[4,5,10,11]$.

\section{Conclusions}

In the present article the anatomical landmarks that define the cervical lymphatic spaces as they are identified by ultrasonography are illustrated. The use of these landmarks allows the topographic localization of the cervical lymph nodes, with major implications in the staging of various conditions and in adequate treatment decisions.

\section{Conflict of interest: none}

\section{References}

1. Badea RI, Baciut M. Limfonodulii cervicali. In: Badea RI, Dudea SM, Mircea PA, Zdrenghea D. Tratat de ultrasonografie clinica. Vol 2. Capul si gatul, toracele si mediastinul, ecocardiografie si vasele membrelor. Ed. Medicala, Bucuresti 2006: 133-147.

2. Mircea PA, Has V, Cucuianu A. Splina si sistemul limfatic abdominal. In: Badea RI, Dudea SM, Mircea PA, Stamatian F. Tratat de ultrasonografie clinica, Vol 1. Principii, abdomen, obstetrica si ginecologie. Ed. Medicala, Bucuresti 2007: 393-394.

3. Ying MTC, Ahuja AT. Ultrasonography of cervical lymph nodes. www.droid.cuhk.edu.hk.

4. Ruggiero FP. Neck dissection classification. www.emedicine.medscape.com.

5. Baciut M. Ecografia afectiunilor chirurgicale maxilofaciale. Ed Sincron, Cluj-Napoca 2002: 164-176.

6. Rouvière H. Lymphatic system of the head and neck. Ann Arbor, MI: Edwards Brothers, 1938.

7. Shah JP, Strong E, Spiro RH, Vikram B. Surgical grand rounds: neck dissection: current status and future possibilities. Clin Bull 1981; 11: 25-33.

8. Fleming ID, Cooper JS, Henson DE, et al. American Joint Committee on Cancer Staging manual, 5th ed., Philadelphia: Lippincott Raven, 1997.

9. Hajek PC, Salomonowitz E, Turk R, Tscholakoff D, Kumpan W, Czembirek H. Lymph nodes of the neck: evaluation with US. Radiology 1986; 158: 739-742.

10. Ahuja AT, Evans R. (Eds.). Practical head and neck ultrasound. Greenwich Medical Media, London 2000; 1: 67-70.

11. Ying M, Ahuja AT. Sonography of neck lymph nodes. Part I: normal lymph nodes. Clin Radiol 2003; 58: 351-358. 